\title{
Application of Ontology Technology in Agricultural Information Retrieval
}

\author{
Jincui Kang \\ Department of Computer, \\ Shijiazhuang University \\ Shijiazhuang,050035, China \\ E-mail:Liuzhiguolunwen@163.com
}

\author{
Jinglong Gao \\ Department of Social Sciences Education, \\ Hebei Institute Physical Education \\ Shijiazhuang,050035,China \\ E-mail:Lxn8001@163.com
}

\begin{abstract}
The agricultural information on the internet become more and more, it is very difficult to search accurate related information from such different information, in order to improve the efficiency of information retrieval on the internet, the intelligent searching technology of agricultural information based on ontology is proposed. The paper firstly introduces research on the agricultural ontology and information retrieval, and takes agriculture domain knowledge as research object, analyzes the characters of agricultural domain knowledge and semantics retrieval, then uses the agricultural ontology to make the structure of agriculture ontology knowledge, and constructs the related agricultural knowledge ontology and knowledge base, implementing the intelligent searching of the agricultural information. The results indicate that the application of agricultural ontology technology in the agricultural information retrieval not only achieves the intelligent retrieval of agricultural information, but also greatly improves the accuracy and reliability of agricultural information retrieval.
\end{abstract}

Keywords- ontology; agricultural knowledge; retrieval

\section{INTRODUCTION}

Agricultural ontology is the scientific methodology that the computer language is used to regulate the concept expression of agricultural knowledge, then the organization of agricultural knowledge is made and agricultural knowledge services are developed. In china the agricultural ontology is becoming research hot of agricultural information domain, which has become new direction of knowledge engineering in agriculture. The research on agricultural ontology can contribute to organization and invention of agricultural scientific knowledge and provide theory and methods for organization of agricultural knowledge, and lay foundation for further agricultural research and application of semantic web. Meanwhile the conditions are made to apply agricultural information in data mining, knowledge representation, automatic indexing, information classification, intelligent retrieval, multilingual translation, knowledge discovery and application of research in the field, then the cooperation between domestic and international agricultural will be promoted in the field of ontology research, and the close cooperation of agricultural experts and IT experts will enhance overall level of ontology in the field of agricultural research level, so catch up with international standards.

The research and development of key technology on agricultural knowledge and semantic retrieve will provide knowledge retrieval technology based on agriculture domain ontology, and the single platform is used to access a variety of knowledge resources and to carry out the organization, mining[1], the retrieval and expression of agriculture, agricultural knowledge service which belongs to one or multiple is used to be made, which can greatly improve the accuracy and reliability of agricultural information retrieval.

\section{RELATED RESEARCH}

\section{A. Agricultural Knowledge}

Agricultural knowledge consists of structural agricultural experience and research habits, values concept, related information and expert knowledge, and agricultural knowledge not only are shown in the file or library, but also are included in the agricultural production and research of everyday life, work, procedures, practices, case studies and norms. The agricultural knowledge are action-oriented, and are made dynamic organization and expression by the requirement of user and driving programs, and which can help people directly engage in agricultural production and business activities. Agriculture knowledge includes explicit knowledge and tacit knowledge, the explicit knowledge is easy to be saved and distributed by the information technology, and the tacit knowledge usually are the experience, skills and sixth sense of intuition, and sometimes only means that are not conveyed in words, which are dominant outsider, and which requires people make the formal code, the information technology is used to reach external storage and transmission, sharing and widely application of agricultural, the agricultural knowledge are different from the agricultural information, information and knowledge are interrelated and interdependent, but are very distinct[2].

\section{B. Semantic Retrieval}

The semantic retrieval of agricultural knowledge is not only single point urgent need of agricultural information service, but further is the premise and foundation of distributed knowledge grid environment semantic retrieval. 
Semantic retrieval of agricultural knowledge can not simply rely on semantic search technology literature resources, rapidly growing network of information resources and vast spatial information resource, meanwhile are also important information resources for semantic retrieval. However these resources have not been effective organized and achieved integration of information resources and technology.

Searching strategy is an important factor of affecting searching results, then many scholars have proposed a variety of intelligent searching models, but a wide range of applications are used to be confirmed. As a variety of information resources which the search service depends on are dynamically updated, and the retrieval system of selflearning, self-maintenance need to be built. To achieve intelligent knowledge retrieval, we not only require human thinking process of inference, but also require the strong support of ontology. Obviously at home and abroad these research areas have not yet achieved satisfactory results and application examples. Semantic retrieve of knowledge for agriculture is an exploratory research and cutting-edge work, which will have great effect on agriculture and development of semantic web[3].

\section{Agricultural Ontology}

Agricultural ontology can be defined as recognized and formal specification on concept system in agriculture science. Ontology is the basis of formal description which involves important domain of entities, attributes, processes and mutual relations, which can achieve interoperability and inheritance of different systems; and can also provide a basic structure for the construction of knowledge base. The construction of agricultural ontology will contribute to common understanding of the organizational structure and understanding and analysis of agricultural knowledge, and lay the foundation for semantic networks. The use of ontological methods can improve the organization and integration of information resources, knowledge searching, knowledge accumulation, knowledge sharing, the efficiency of knowledge services, knowledge reuse and knowledge sharing will become reality[4].

The establishment of agricultural ontology is the basis of intelligent searching of agricultural information, which can reintegrate existing resources and regulate new resources, find the internal logic of relations of resources, then this intelligent search of agricultural information services is made, and will contribute to agricultural knowledge management and knowledge innovation, and radically improve service quality and technical level of agricultural information, so a large number of agricultural information resources which exit in variety of media and agricultural research institutions, agricultural universities and other institutions are efficiently used, and provide service for the real customer. Therefore the generic searching tool based on the existing relevance and accuracy of search results are difficult to meet the needs of agricultural information on the internet, and the heterogeneous information resources in agricultural areas are not made full use. The research and development in the agricultural sector based on information intelligent search technology and construction of agricultural information for the professional search tool will become important agricultural information technology[5].

\section{Research on Knowledge Ontology and Engine of Agricultural Information}

\section{1) Research of agricultural information knowledge ontology:}

- Construction of agricultural knowledge ontology and research on storage technology are made: The help is from waterfall model of ontology construction, agricultural product technology ontology and agricultural product commodities markets ontology are constructed based on the existing agricultural science technology literature of ontology, the concept system of standardization of agricultural knowledge is made, and all the logical relationships between concepts and attributes are determined, which provide prerequisite for intelligent searching of knowledge organization and information agricultural. And the research on ontology relational database storage technology are made, and the ontology concepts, attributes, relationships are stored in relational database by a certain structure, and export ontology according as XML format, in order to facilitate the exchange between the ontology and mapping, and perform research of ontology application[5].

- Research on ontology interoperability technology of agricultural underlying is made: The underlying ontology interoperability technology of agricultural production technology ontology, agricultural product ontology markets, agricultural commodities ontology and existing agricultural science and technology are made to enable the ontology to access each other and pass messages to implement the different types of knowledge, information linkages, mutual visits, effectively improve the retrieval recall rate.

- Research on construction of agricultural knowledge base technology is made. The knowledge base of agricultural technology are studied, which are used to extract information and regulate building process of agricultural production technology knowledge base, knowledge base of agricultural products and commodities market knowledge base, and provide unified agricultural intelligent retrieval platform for agricultural areas.

2) Research of agricultural information knowledge engine:

- Research on background model and individual knowledge model based on ontology are made: The knowledge model based on information resource requirement is studied, which includes the background level of models, relationships, mapping, conversion mechanisms. The model and its XML representation mechanism and operation are studied. The ontology information resource requirement of those who can represent some aspects of structure of 
formal knowledge representation model is studied. The evolutionary relationships of individual knowledge and background knowledge, as well as individual knowledge model refinement, conversion, mapping operation relations. Knowledge model of individual expression and its mechanism of XML operations are studied [5].

- Construction of agricultural knowledge engine is made: The research on ontology background knowledge, individual knowledge model and architecture of knowledge engine model of agricultural are made, the matching and refinement mechanism on background ontology and individual knowledge are analyzed, which are used to determine the knowledge engine, then achieve model matching and matching algorithms and the calculation of the specific implementation. A typical model for the formation of background knowledge and individual knowledge model library database are built, and continue to enrich and be perfect. Knowledge of engine performance is analyzed and the techniques and methods that enhance the performance are studied.

- Adaptation service of knowledge engine and searching engine are made: The determination of the knowledge engine interface specification is performed, and the mainstream general searching engine interface is analyzed, so the collection of adapter service interface specification is performed. The knowledge model for analysis of the individual general searching engine are used to meet the specific set of words and sent to the appropriate knowledge of general searching engines adapter service. The adaptive service which is oriented in agricultural areas and specification professional intelligent searching services which includes knowledge of engines, general searching engines are developed.

\section{APPLICATION OF AGRICULTURAL ONTOLOGY}

The ontology plays an important role on the information retrieval, the called knowledge retrieve is that the advanced intelligent theory and application of technology are used to make a series of semantic analysis on information resources and user questions. The deeper meaning is explored, so that we can accurately express knowledge resources and user requirements, and then search in various heterogeneous database, data warehouse and knowledge base, then after intelligent information processing is made, the relevant results of the retrieval mechanism in this ontology are returned, the retrieval system workflow of ontological knowledge generally consists of the following four steps:

\section{A. Establishment of Domain Knowledge of Agricultural Ontology}

The standardization description of ontology concepts and their interrelationships of agricultural information are made, basic knowledge system and description language of particular domain are constructed, and lay the foundation for achievement of some degree of knowledge organization, knowledge reuse, retrieval and sharing.

\section{B. Establishment of Agricultural Ontology}

The ontology provides a set of terms and concepts to describe some field of agricultural information, the object library uses the ontology to express these terms in the field of facts and knowledge. The establishment of ontology knowledge base is to collect the information source data, with reference to the established ontology metadata model, all kinds of heterogeneous will be unified into a unified document format, and make restructuring and integration, which can solve the problems of heterogeneous nature.

\section{Document Classification}

Document classification is the core of ontology base construction, the summary document of key words and content are listed in the majority of retrieval documents, we can use them as the main information, and with the help of the ontology we can determine which category the document belongs to, then we can carry out this classification of the document. The ontology object library is a dynamic process, in order to keep pace with the ontology library and information resources, the system retrieves the introduction of intelligent agents in adaptive learning, when the retrieval agent in system service is small, the statistical user makes preference ontology library content, access user interest trends, and then take the initiative to build a searching query and submit to the search agent, then the searching agent can access the information resources, get new documents of related fields, and then the ontology field agents are used to confirm with the requirements of division, the format is unified to sort according to importance, and the corresponding results are stored in the ontology library.

\section{Knowledge Retrieval Process}

The knowledge retrieve process include the standardization of user searching and retrieve requests processing stages, the standardization of user searching requests is that the retrieve system creates a personalized retrieve request which fits the requirement of user, and makes the user complete the construction of ontology under the guidance of retrieval-style building. The process and knowledge of searching results are returned to the searching results to extract the user interface agent, the use of domain ontology knowledge is made to carry out the analysis of searching results, filter, conversion, classification and integration of learning and knowledge extraction.

\section{SIMULATION RESULTS AND DISCUSSION}

\section{A. Simulation}

When the user inputs the searching terms, the system will display the classification of information which are related to this word, the user can make further options in the classified information, then obtain accurate information. Intelligent retrieval can apply key words and the class number group in position searching, which improves the speed of queries from the original literature database, and the users can have 
comprehensive understanding of the database information about the distribution, and find the information they need in order to avoid a large number of data records which are kept by the page to find, so reduce the interaction with the remote server during the visiting. The comparison of intelligent retrieval methods and the conventional methods are shown in Table 1:

TABLE I. THE COMPARISON OF TWO METHODS

\begin{tabular}{|c|c|c|c|}
\hline \multicolumn{2}{|c|}{ Traditional methods } & \multicolumn{2}{|c|}{ Improved methods } \\
\hline Accuracy & Recall & Accuracy & Recall \\
\hline 81 & 82 & 89 & 94 \\
82 & 83 & 91 & 93 \\
83 & 84 & 89 & 92 \\
81 & 85 & 89 & 93 \\
85 & 84 & 89 & 89 \\
81 & 87 & 88 & 89 \\
82 & 88 & 87 & 88 \\
78 & 86 & 89 & 87 \\
77 & 85 & 87 & 89 \\
76 & 89 & 89 & 87 \\
75 & 90 & 78 & 89 \\
79 & 76 & 85 & 78 \\
90 & 78 & 98 & 85 \\
92 & 79 & 89 & 98 \\
75 & 79 & 90 & 89 \\
77 & 70 & 90 & 90 \\
& & & \\
\hline
\end{tabular}

\section{B. Discussion}

It can seen from the Table 1 that the recall rate increases from $75.6 \%$ to $88.5 \%$, which is higher than the conventional retrieval under the same conditions, and the precision rate of is more than $89 \%$, which is also significantly better than the conventional retrieval precision. In short, the system fully considers the thinking way of people, reflects the level of structure in the organization of agricultural information, so the efficiency of user clicks is improved, and the relationship between the concepts which are described by ontology is applied to the retrieve of information, especially while the user is not certain for queries target, it can better reflect the intelligence analysis of user requirement and retrieval results effect.

\section{CONCLUSION}

The research on agricultural ontology construction technology will contribute to the ontology establishment of different domains in agriculture, and gradually build larger domain agricultural ontology, which can develop from the senior or junior ontology to the heavy weight ontology, and lay foundation for information resources of the network organization and construction of next-generation semantic web, making the organization of agricultural information resources, organizing information into knowledge from the organization, so the research on the ontology has general scientific significance and practical value.

\section{ACKNOWLEDGMENT}

We are very grateful to Professor Zhang for his kind assistance and discussion. And we also thank for the support from China Agricultural University.

\section{REFERENCES}

[1] Y.Xue,D.Y. Ye,W.D Zhang. The Research on Saurus Construction of Domain Ontology China Classification. Journal of Intelligence, vol3, pp.16-18,2007.

[2] A.M Tang, Q.Zhen,J.Fan. The Saurus Approach to Build Domain Ontology. New Technololgy of Library and Information Service, vol4, pp.1-5,2005.

[3] N.F.Xie,W.S.Wang. Construction Method of Agricultural Knowledge Ontology. Agricultural Network Information, vol28, pp.12-16,2008.

[4] W.H.Du.Comparative Study of Ontology Construction Method. Information Science, vol10, pp.24-25,2005.

[5] G.X.He, J.X.Wang,Z.G Cang. Design and Realization of Vegetable Pathological Aid Diagnosis System.Journal of Shandong Agricultural University: Natural Science,vol38,pp.58-63,2007. 